\title{
Novel Furanoid $\alpha$-Substitued $\alpha$-Amino Acid as a Potent Turn Mimic in Peptide Synthesis
}

\section{Miroslava Martinková *, Jozef Gonda and Jana Raschmanová}

Institute of Chemical Sciences, Department of Organic Chemistry, P. J. Šafárik University, Moyzesova 11, SK-040 01 Košice, Slovak Republic. Tel.: (+ 421) 556228332; Fax: (+ 421) 556222421

E-mails: Jozef Gonda: jgonda@kosice.upjs.sk, Jana Raschmanova: jrasch@pobox.sk

* Author to whom correspondence should be addressed; e-mail: mmartin@kosice.upjs.sk

Received: 23 June 2006; in revised form: 20 July 2006 / Accepted: 21 July 2006 / Published: 26 July 2006

\begin{abstract}
A stereoselective approach has been developed to the new sugar amino acid and potential potent turn mimic 5-O-(tert-butyldimethylsilyl)-3-deoxy-1,2-Oisopropylidene-3-methoxycarbonylamino- $\alpha$-D-xylofuranose 3-C-carboxylic acid (12), via the [3,3]-sigmatropic rearrangement of allylic thiocyanates $(Z)-6$ and $(E)-7$, prepared from D-xylose. The synthesis of a new dipeptide $\mathbf{1 3}$ is also described.
\end{abstract}

Keywords: Sugar amino acids, stereoselectivity, sigmatropic rearrangement, carbohydrate-based mimetics, peptidomimetic, $\beta$-turn mimic

\section{Introduction}

Sugar amino acids (SAAs) can be found in nature largely as a molecules that combine the structural features of simple amino acids with those of simple carbohydrates [1]. The resulting hybrid is a highly substitued polyfunctionalized synthon which can be used for the preparation of modified analogues of biologically active peptides and/or oligosaccharides. Sugar amino acids represent an important class of conformationally constrained templates that have been used extensively in recent years in many peptidomimetic studies [1, 2] and have emerged as attractive building blocks for the incorporation of a sugar moiety into short peptide sequences using standard peptide coupling techniques, thus opening the door to novel peptidomimetics. They are also known as $\beta$-turn mimics 
and introduction of conformationally restricted nonpeptide isosteres into the peptide backbone to achieve desirable secondary structures is a great interest of many synthetic chemists [3].

\section{Results and Discussion}

We report herein a synthetic approach to 5-O-(tert-butyldimethylsilyl)-3-deoxy-1,2-Oisopropylidene-3-methoxycarbonylamino- $\alpha$-D-xylofuranose 3 -C-carboxylic acid (12) as a building block for peptide scaffold and conformationally restrained peptidomimetics. The Wittig reaction of known 5-O-TBDMS-1,2-O-isopropylidene- $\alpha$-D-erytro-pentofuranos-3-ulose (1) [4] with ethoxycarbonylmethyl-enetriphenylphosphorane in dry dichloromethane gave, after chromatographic separation, pure $(Z)-\alpha, \beta$-unsaturated ester 2 and its $(E)$-isomer 3 (9.6:1, 90.5\%) (Scheme 1$)$. The $Z$ and $E$ configurations of the exocyclic double bond in $\mathbf{2}$ and $\mathbf{3}$ were determined by ${ }^{1} \mathrm{H}-\mathrm{NMR}$ spectral analysis, including NOE data. The irradiation of the $\mathrm{H}-4$ proton in 2 led to a $3.4 \%$ enhancement of the intensity of H-6, while the irradiation of the H-6 proton resulted in a $3.9 \%$ enhancement of the intensity of the $\mathrm{H}-4$ signal, along with a small $1.3 \%$ enhancement of the $\mathrm{H}-2$ proton. On the other hand, when the $\mathrm{H}-2$ proton in 3 was irradiated, a strong (5.0 \%) NOE enhancement of the H-6 signal was observed, while the irradiation of the H-6 proton resulted in a $4.2 \%$ enhancement of the intensity of the H-2 signal. Finally, the irradiation of the H-4 proton in 3 resulted in a $1.2 \%$ enhancement of the intensity of the H-6 proton signal. Reduction of esters with $\mathrm{LiAlH}_{4}$ in dry diethyl ether afforded the allylic alcohols (Z)-4 and (E)-5 in 96 and 72\% yields, respectively, after silica-gel chromatography. The thiocyanates (Z)-6 and (E)-7 were prepared by $\mathrm{S}_{\mathrm{N}} 2$ substitution of the $O$-mesyl group in the corresponding mesylates, derived from allylic alcohols $(Z)-\mathbf{4}$ and $(E)-\mathbf{5}$, by the thiocyanate group ( $\mathrm{KSCN} / \mathrm{CH}_{3} \mathrm{CN}$ ) (Scheme 1).

\section{Scheme 1.}

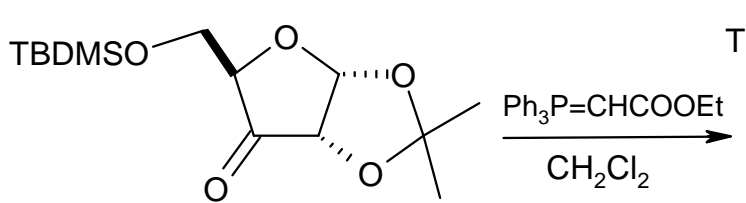

$\underline{1}$

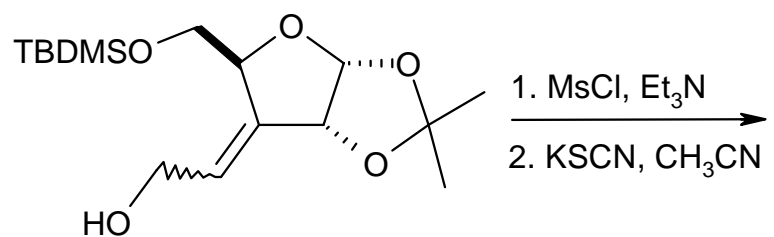

(Z)-4, 96\%

(E) $\underline{-}, 72 \%$

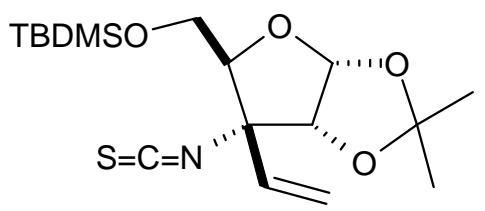

$\underline{8 a}$

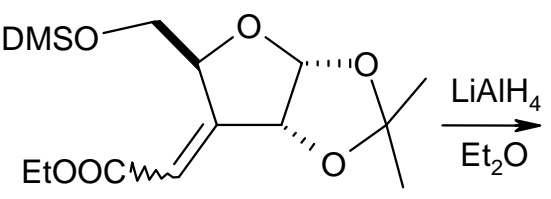

(Z)-2, 82\%

(E)- $\underline{3}, 8.5 \%$

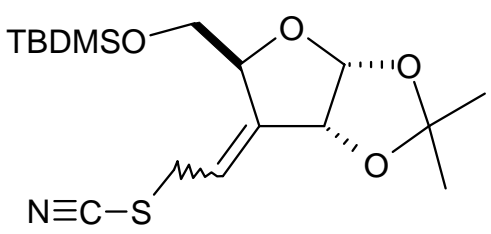

(Z)- $\underline{\mathbf{6}}, 73 \%$

(E) $-\underline{\mathbf{z}}, 70 \%$

$\downarrow$ heptane, $90^{\circ} \mathrm{C}$,

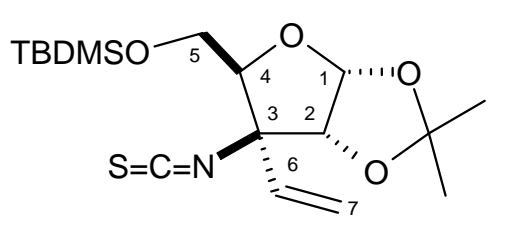

8 
The thermal rearrangements of thiocyanates (Z)-6 and (E)-7 was carried out in heptane under a $\mathrm{N}_{2}$ atmosphere at $90{ }^{\circ} \mathrm{C}$ for $16 \mathrm{~h}$ and gave isothiocyanate 8 as the sole reaction product in 82 and $70 \%$ yields, respectively, after silica-gel chromatography. The epimeric isothiocyanate 8a was not detected in the reaction mixtures. The stereochemistry of the new quarternary carbon center (C-3) introduced in 8 was determined as $(S)$ by ${ }^{1} \mathrm{H}-\mathrm{NMR}$ spectral analysis, including NOE data. Thus, when the H-4 proton was irradiated, a strong (8.2\%) enhancement of the intensity of the H-6 signal was noted and the irradiation of the H-6 proton resulted in a $5.4 \%$ enhancement of the intensity of the $\mathrm{H}-4$ signal, indicating a cis relationship between the vinyl group and the $\mathrm{H}-4$ proton on the furanoside ring (Figure 1).

\section{Figure 1}

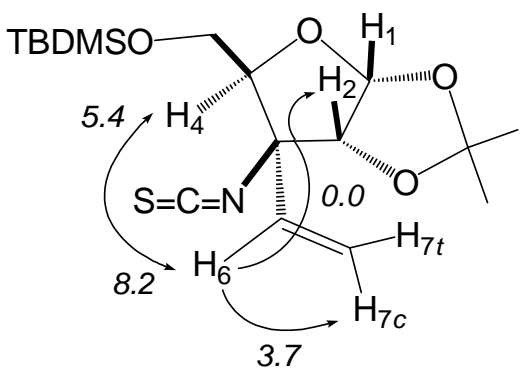

In our subsequent strategy, the isolated isothiocyanate $\mathbf{8}$ was converted into the desired $\alpha$ substitued $\alpha$-amino acid 12. In the first step, the isothiocyanate group was transformed into the thiourethane 9 in 95\% yield after silica-gel chromatography by its reaction with $\mathrm{CH}_{3} \mathrm{ONa}$ in dry methanol at room temperature for $4 \mathrm{~h}$. The thus prepared thiocarbamate $\mathbf{9}$ was converted into the corresponding oxygen derivative $\mathbf{1 0}$ by the action of mesitylnitrile oxide in acetonitrile (23 h, 93\%) (Scheme 2).

\section{Scheme 2.}

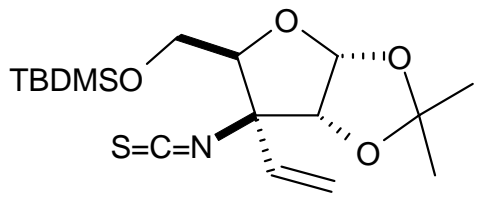

$\underline{8}$

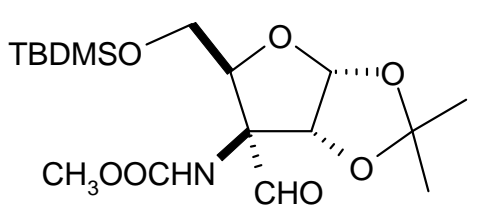

$\underline{11}$

$\downarrow \begin{aligned} & \mathrm{NaClO}_{2} \\ & 74 \%\end{aligned}$

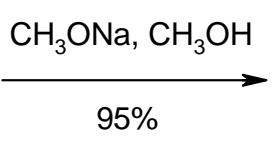

$\frac{\mathrm{NaIO}_{4}, \mathrm{RuCl}_{3}}{70 \%}$<smiles>C=C[C@]1(NC(=S)OC)C(COCC(C)C)O[C@@H]2OC(C)(C)O[C@H]21</smiles>

$\underline{9}$

$\downarrow \begin{gathered}\mathrm{MNO}, \mathrm{CH}_{3} \mathrm{CN} \\ 93 \%\end{gathered}$<smiles>C=C[C@]1(NC(=O)OC)C(COC(C)C)O[C@@H]2OC(C)(C)OC2[C@H]1C</smiles>

$\underline{10}$<smiles>COC(=O)N[C@@]1(C(=O)O)C(COS(C)(C)C)O[C@@H]2OC(C)(C)O[C@H]21</smiles>
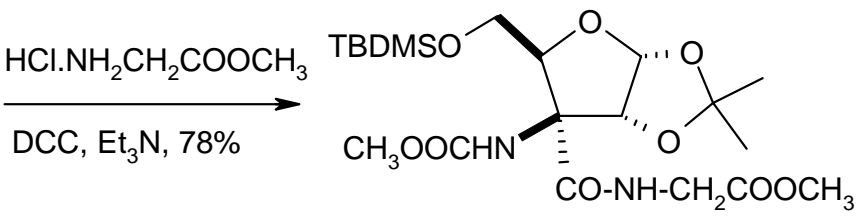
The oxidation of carbamate $\mathbf{1 0}$ was accomplished with a catalytic amount of ruthenium (III) chloride and $\mathrm{NaIO}_{4}$ in 2:2:3 $\mathrm{CCl}_{4} / \mathrm{CH}_{3} \mathrm{CN} / \mathrm{H}_{2} \mathrm{O}$ to give aldehyde 11 in $70 \%$ yield. This aldehyde was then oxidized to the protected amino acid 12 in 74\% yield (after flash chromatography) by treatment with sodium chlorite/2-methyl-2-butene (Scheme 2).

Among the methods available for the linkage of amino acids and peptides coupling with carbodiimides is one of the most frequently used. Our coupling reaction with DCC [5] and glycine methyl ester hydrochloride was performed in dry dichloromethane in the presence of $\mathrm{Et}_{3} \mathrm{~N}$ at $0{ }^{\circ} \mathrm{C}$ for 1 $\mathrm{h}$ and then for $18 \mathrm{~h}$ at room temperature to afford dipeptide $\mathbf{1 3}$ in 78\% yield after silica-gel chromatography (Scheme 2).

\section{Conclusions}

In summary, a stereocontrolled synthesis of the nonproteinogenic $\alpha$-substitued $\alpha$-amino acid $\mathbf{1 2}$ employing thiocyanates $(Z)-\mathbf{6}$ and $(E)-\mathbf{7}$ as the educts has been reported. The coupling reaction between this sugar amino acid $\mathbf{1 2}$ and glycine methyl ester provided dipeptide $\mathbf{1 3}$ as a potent peptidomimetic.

\section{Experimental}

\section{General}

The melting points were determined on the Kofler block and are uncorrected. Optical rotations were measured in chloroform with a P3002 Krüss polarimeter and reported as follows: $[\alpha]_{\mathrm{D}}{ }^{25}$ ( $c$ in $\mathrm{g}$ per $100 \mathrm{~mL}$ ). NMR spectra were recorded at room temperature on a Varian Mercury Plus 400 FT NMR spectrometer $\left({ }^{1} \mathrm{H}\right.$ at $400.13 \mathrm{MHz}$ and ${ }^{13} \mathrm{C}$ at $\left.100.6 \mathrm{MHz}\right)$. Chemical shifts are referenced either to tetramethylsilane used as internal standard for ${ }^{1} \mathrm{H}$ or to the solvent signal $\left({ }^{13} \mathrm{C}-\mathrm{NMR}, \delta \mathrm{CDCl}_{3}=77.0\right)$. ${ }^{13} \mathrm{C}$-NMR multiplicities were determined by using a DEPT pulse sequence. Reactions were routinely monitored by TLC (Merck $60 \mathrm{~F}_{254}$ ) and the products were visualized by UV light absorption at $254 \mathrm{~nm}$ or by spraying with Mo-reagent or $\mathrm{KMnO}_{4}$-reagent. All reactions were performed under an atmosphere of nitrogen. Solvents were purified by standard procedures and distilled before use. Column chromatography was carried out on the glass columns using Kieselgel (0.035-0.070 mm) silica gel. 5-O-(tert-Butyldimethylsilyl)-1,2-O-isopropylidene- $\alpha$-D-erythro-pentofuranosyl-3-ulose (1) was prepared according to a published procedure [4].

5-O-(tert-Butyldimethylsilyl)-3-C-(Z)-carboethoxymethylene-3-deoxy-1,2-O-isopropylidene- $\alpha$-D-xylofuranose (2) and its (E)-isomer (3)

(Carboethoxymethylene)triphenylphosphorane (7.84 g, $22.4 \mathrm{mmol}$ ) was added to a solution of ketone 1 [4] (6.18 g, $20.4 \mathrm{mmol})$ in dry $\mathrm{CH}_{2} \mathrm{Cl}_{2}(80 \mathrm{~mL})$. The reaction mixture was stirred at room temperature for $16 \mathrm{~h}$. The solvent was removed under reduced pressure and the residue was purified by chromatography on silica gel (hexane-ethyl acetate, 13:1) to afford (E)-3 (0.65 g, 8.5\%) and (Z)-2 $(6.21 \mathrm{~g}, 82 \%)$ as colorless oils. Compound 3: $[\alpha]_{\mathrm{D}}{ }^{25}=+233.9(c \mathrm{0.20})$; ${ }^{1} \mathrm{H}-\mathrm{NMR}: \delta-0.01\left(3 \mathrm{H}, \mathrm{s}, \mathrm{CH}_{3}\right)$, 
0.02 (3H, s, $\mathrm{CH}_{3}$ ), 0.86 (9H, s, 3 x CH $\left.\mathrm{CH}_{3}\right), 1.30$ (3H, t, $J=7.2 \mathrm{~Hz}, \mathrm{CH}_{3}$ ), 1.40 (3H, bs, $\mathrm{CH}_{3}$ ), 1.43 (3H, bs, $\left.\mathrm{CH}_{3}\right), 3.78\left(1 \mathrm{H}, \mathrm{dd}, J_{5,5}=10.4 \mathrm{~Hz}, J_{5,4}=2.2 \mathrm{~Hz}, \mathrm{H}_{5}\right), 3.93\left(1 \mathrm{H}, \mathrm{dd}, J_{5,5}=10.4 \mathrm{~Hz}, J_{5,4}=1.6 \mathrm{~Hz}, \mathrm{H}_{5}\right), 4.19$ $\left(2 \mathrm{H}, \mathrm{q}, J=7.2 \mathrm{~Hz}, \mathrm{CH}_{2} \mathrm{O}\right), 5.03\left(1 \mathrm{H}, \mathrm{ddd}, J_{2,1}=4.7 \mathrm{~Hz}, J_{2,4}=1.8 \mathrm{~Hz}, J_{6,2}=1.8 \mathrm{~Hz}, \mathrm{H}_{2}\right), 5.57-5.59(1 \mathrm{H}, \mathrm{m}$, $\left.\mathrm{H}_{4}\right), 5.95\left(1 \mathrm{H}, \mathrm{d}, J_{2,1}=4.7 \mathrm{~Hz}, \mathrm{H}_{1}\right), 6.13\left(1 \mathrm{H}, \mathrm{dd}, J_{6,4}=1.8 \mathrm{~Hz}, J_{6,2}=1.8 \mathrm{~Hz}, \mathrm{H}_{6}\right) ;{ }^{13} \mathrm{C}-\mathrm{NMR}: \delta-5.7,-5.5$, 14.2, 18.2, 25.9 (3 x C), 27.7, 27.8, 60.5, 65.8, 82.4, 82.6, 104.5, 113.2, 116.2, 160.8, 165.5; Anal. Calcd for $\mathrm{C}_{18} \mathrm{H}_{32} \mathrm{O}_{6} \mathrm{Si}$ (372.54): C 58.03, H 8.66; found C 57.91, H 8.78. Compound 2: $[\alpha]_{\mathrm{D}}{ }^{25}=+131.3$ (c 0.20); ${ }^{1} \mathrm{H}-\mathrm{NMR}: \delta 0.05$ (3H, s, $\left.\mathrm{CH}_{3}\right), 0.06\left(3 \mathrm{H}, \mathrm{s}, \mathrm{CH}_{3}\right), 0.88\left(9 \mathrm{H}, \mathrm{s}, 3 \times \mathrm{CH}_{3}\right), 1.30(3 \mathrm{H}, \mathrm{t}, J=7.1 \mathrm{~Hz}$, $\left.\mathrm{CH}_{3}\right), 1.43\left(3 \mathrm{H}, \mathrm{bs}, \mathrm{CH}_{3}\right), 1.48\left(3 \mathrm{H}, \mathrm{bs}, \mathrm{CH}_{3}\right), 3.74\left(1 \mathrm{H}, \mathrm{dd}, J_{5,5}=10.7 \mathrm{~Hz}, J_{5,4}=3.5 \mathrm{~Hz}, \mathrm{H}_{5}\right), 3.79(1 \mathrm{H}$, dd, $\left.J_{5,5}=10.7 \mathrm{~Hz}, J_{5,4}=4.3 \mathrm{~Hz}, \mathrm{H}_{5}\right), 4.25\left(2 \mathrm{H}, \mathrm{q}, J=7.1 \mathrm{~Hz}, \mathrm{CH}_{2} \mathrm{O}\right), 4.84-4.87\left(1 \mathrm{H}, \mathrm{m}, \mathrm{H}_{4}\right), 5.64-5.66$ $\left(1 \mathrm{H}, \mathrm{m}, \mathrm{H}_{2}\right), 5.91\left(1 \mathrm{H}, \mathrm{d}, J_{1,2}=4.1 \mathrm{~Hz}, \mathrm{H}_{1}\right), 6.01\left(1 \mathrm{H}, \mathrm{dd}, J_{6,2}=1.7 \mathrm{~Hz}, J_{6,4}=1.7 \mathrm{~Hz} \mathrm{H}\right)_{6}$; ${ }^{13} \mathrm{C}-\mathrm{NMR}: \delta$ 5.6, -5.5, 14.1, 18.1, 25.7(3 x C), 27.2, 27.5, 60.5, 65.3, 78.8, 81.0, 105.4, 112.7, 116.5, 156.8, 164.9; Anal. Calcd for $\mathrm{C}_{18} \mathrm{H}_{32} \mathrm{O}_{6} \mathrm{Si}$ (372.54): C 58.03, H 8.66; found C 58.15, H 8.55.

5-O-(tert-Butyldimethylsilyl)-3-deoxy-3-C-(E)-(2-hydroxyethylidene)-1,2-O-isopropylidene- $\alpha$-D-xylofuranose (5)

$\mathrm{LiAlH}_{4}(0.045 \mathrm{~g}, 1.18 \mathrm{mmol})$ was added at $0{ }^{\circ} \mathrm{C}$ to a solution of $(E)-3(0.44 \mathrm{~g}, 1.18 \mathrm{mmol})$ in dry $\mathrm{Et}_{2} \mathrm{O}(7.2 \mathrm{~mL})$. The reaction mixture was stirred at $0{ }^{\circ} \mathrm{C}$ for $15 \mathrm{~min}$ and then for $1.5 \mathrm{~h}$ at room temperature. The reaction was quenched by careful addition of water $(0.3 \mathrm{~mL})$ and the precipitate was removed by filtration. The filtrate was dried $\left(\mathrm{Na}_{2} \mathrm{SO}_{4}\right)$ and concentrated under reduced pressure. Chromatography of the residue (hexane-ethyl acetate, 3:1) afforded $0.28 \mathrm{~g}$ (72 \%) of allylic alcohol 5 as a white solid; m.p. $42-43{ }^{\circ} \mathrm{C}$; $[\alpha]_{\mathrm{D}}{ }^{25}=+79.6$ (c 0.20); ${ }^{1} \mathrm{H}-\mathrm{NMR}$ : $\delta-0.01\left(3 \mathrm{H}, \mathrm{s}, \mathrm{CH}_{3}\right), 0.06(3 \mathrm{H}$, s, $\left.\mathrm{CH}_{3}\right), 0.88$ (9H, s, $\left.3 \times \mathrm{CH}_{3}\right), 1.41$ (3H, bs, $\left.\mathrm{CH}_{3}\right), 1.45$ (3H, bs, $\left.\mathrm{CH}_{3}\right), 2.18-2.30(1 \mathrm{H}, \mathrm{m}, \mathrm{OH}), 3.68(1 \mathrm{H}$, dd, $\left.J_{5,5}=10.8 \mathrm{~Hz}, J_{5,4}=4.0 \mathrm{~Hz}, \mathrm{H}_{5}\right), 3.72\left(1 \mathrm{H}, \mathrm{dd}, J_{5,5}=10.8 \mathrm{~Hz}, J_{5,4}=2.9 \mathrm{~Hz}, \mathrm{H}_{5}\right), 4.16-4.18\left(2 \mathrm{H}, \mathrm{m}, \mathrm{H}_{7}\right)$, 4.95-4.97 (1H, m, $\left.\mathrm{H}_{2}\right)$, 5.05-5.09 $\left(1 \mathrm{H}, \mathrm{m}, \mathrm{H}_{4}\right), 5.84\left(1 \mathrm{H}, \mathrm{d}, J_{2,1}=4.4 \mathrm{~Hz}, \mathrm{H}_{1}\right)$, 5.99-6.04 $\left(1 \mathrm{H}, \mathrm{m}, \mathrm{H}_{6}\right)$; ${ }^{13}$ C-NMR: $\delta$-5.6, -5.4, 18.3, 25.8 (3 x C), 27.6, 27.9, 60.1, 66.1, 80.2, 82.4, 104.5, 112.8, 126.6, 140.8; Anal. Calcd for $\mathrm{C}_{16} \mathrm{H}_{30} \mathrm{O}_{5} \mathrm{Si}$ (330.50): C 58.15, H 9.15; found C 58.25, H 9.05.

5-O-(tert-Butyldimethylsilyl)-3-deoxy-3-C-(Z)-(2-hydroxymethylidene)-1,2-O-isopropylidene- $\alpha-D-$ xylo-furanose (4)

To a solution of $(Z)-2$ (6.03 g, $16.2 \mathrm{mmol})$ in dry $\mathrm{Et}_{2} \mathrm{O}(100 \mathrm{~mL})$ was added $\mathrm{LiAlH}_{4}(0.61 \mathrm{~g}, 16.2$ mmol) at $0{ }^{\circ} \mathrm{C}$. The reaction mixture was stirred for $15 \mathrm{~min}$ at $0{ }^{\circ} \mathrm{C}$ and then for 1 hour at room temperature. The reaction was quenched with water $(4.2 \mathrm{~mL})$ and the precipitate was removed by filtration. The filtrate was dried $\left(\mathrm{Na}_{2} \mathrm{SO}_{4}\right)$ and concentrated under reduced pressure. Chromatography of the residue (hexane-ethyl acetate, 3:1) gave $5.14 \mathrm{~g}$ (96 \%) of allylic alcohol 4 as a colorless oil; $[\alpha]_{\mathrm{D}}{ }^{25}=+136.3$ (c 0.79); ${ }^{1} \mathrm{H}-\mathrm{NMR}: \delta 0.04\left(3 \mathrm{H}, \mathrm{s}, \mathrm{CH}_{3}\right), 0.05\left(3 \mathrm{H}, \mathrm{s}, \mathrm{CH}_{3}\right), 0.88\left(9 \mathrm{H}, \mathrm{s}, 3 \times \mathrm{CH}_{3}\right), 1.40$ (3H, bs, $\mathrm{CH}_{3}$ ), 1.47 (3H, bs, $\left.\mathrm{CH}_{3}\right), 2.29-2.35(1 \mathrm{H}, \mathrm{m}, \mathrm{OH}), 3.61\left(1 \mathrm{H}, \mathrm{dd}, J_{5,5}=10.6 \mathrm{~Hz}, J_{5,4}=3.4 \mathrm{~Hz}, \mathrm{H}_{5}\right.$ ), $3.74\left(1 \mathrm{H}, \mathrm{dd}, J_{5,5}=10.6 \mathrm{~Hz}, J_{5,4}=3.6 \mathrm{~Hz}, \mathrm{H}_{5}\right), 4.29\left(1 \mathrm{H}, \mathrm{m}, \mathrm{H}_{7}\right), 4.37\left(1 \mathrm{H}, \mathrm{m}, \mathrm{H}_{7}\right) 4.76-4.80\left(1 \mathrm{H}, \mathrm{m}, \mathrm{H}_{4}\right)$, 5.18-5.21 (1H, m, H $)_{2}$, $5.84\left(1 \mathrm{H}\right.$, dddd, $\left.J_{7,6}=6.3 \mathrm{~Hz}, J_{7,6}=6.2 \mathrm{~Hz}, J_{6,2}=1.9 \mathrm{~Hz}, J_{6,4}=1.9 \mathrm{~Hz}, \mathrm{H}_{6}\right), 5.92$ $\left(1 \mathrm{H}, \mathrm{d}, J_{2,1}=4.5 \mathrm{~Hz}, \mathrm{H}_{1}\right)$; ${ }^{13} \mathrm{C}-\mathrm{NMR}: \delta$-5.5, -5.4, 18.2, 25.8 (3 x C), 27.5, 27.6, 60.3, 66.4, 79.1, 81.9, 
105.5, 112.4, 125.9, 141.5; Anal. Calcd for $\mathrm{C}_{16} \mathrm{H}_{30} \mathrm{O}_{5} \mathrm{Si}$ (330.50): C 58.15, H 9.15; found C 58.03, $\mathrm{H}$ 9.25 .

5-O-(tert-Butyldimethylsilyl)-3-deoxy-1,2-O-isopropylidene-3-C-(E)-(2-thiocyanatoethylidene)- $\alpha-D$ xylofuranose (7)

To a solution of $(E)-5(0.23 \mathrm{~g}, 0.70 \mathrm{mmol})$ in dry $\mathrm{CH}_{2} \mathrm{Cl}_{2}(1.7 \mathrm{~mL})$ were added $\mathrm{Et}_{3} \mathrm{~N}(0.15 \mathrm{~mL}, 1.04$ $\mathrm{mmol})$ and $\mathrm{CH}_{3} \mathrm{SO}_{2} \mathrm{Cl}(0.07 \mathrm{~mL}, 0.83 \mathrm{mmol})$ at $0{ }^{\circ} \mathrm{C}$. The reaction mixture was stirred at $0{ }^{\circ} \mathrm{C}$ for 15 $\mathrm{min}$ and then at room temperature for $45 \mathrm{~min}$. The solvent was evaporated under reduced pressure. The residue was diluted with diethyl ether $(3 \mathrm{~mL})$ and the solid was removed by filtration. Evaporation of the solvent under reduced pressure afforded crude mesylate which was used directly in the next reaction without any further purification. To a solution of crude mesylate $(0.27 \mathrm{~g}, 0.66 \mathrm{mmol})$ in $\mathrm{CH}_{3} \mathrm{CN}$ (3 mL), KSCN (0.08 g, $0.82 \mathrm{mmol}$ ) was added. After stirring for $3 \mathrm{~h}$ at room temperature under a nitrogen atmosphere, the solvent was evaporated. The residue was diluted with diethyl ether (3 $\mathrm{mL}$ ) and the solid was removed by filtration. The evaporation of the solvent under reduced pressure and chromatography of the residue (hexane-ethyl acetate, 7:1) afforded $0.18 \mathrm{~g}$ (70\% from 5) of pure thiocyanate 7 as a colorless oil; $[\alpha]_{\mathrm{D}}{ }^{25}=+164.3(c 0.21)$; ${ }^{1} \mathrm{H}-\mathrm{NMR}: \delta 0.05\left(3 \mathrm{H}, \mathrm{s}, \mathrm{CH}_{3}\right), 0.06(3 \mathrm{H}, \mathrm{s}$, $\mathrm{CH}_{3}$ ), 0.88 (9H, s, $3 \times \mathrm{CH}_{3}$ ), 1.42 (3H, bs, $\mathrm{CH}_{3}$ ), 1.47 (3H, bs, $\left.\mathrm{CH}_{3}\right), 3.61-3.67\left(2 \mathrm{H}, \mathrm{m}, \mathrm{H}_{7}, \mathrm{H}_{5}\right), 3.74$ $\left(1 \mathrm{H}, \mathrm{dd}, J_{5,5}=10.6 \mathrm{~Hz}, J_{5,4}=3.8 \mathrm{~Hz}, \mathrm{H}_{5}\right), 3.80\left(1 \mathrm{H}, \mathrm{dd}, J_{7,7}=13.0 \mathrm{~Hz}, J_{7,6}=8.8 \mathrm{~Hz}, \mathrm{H}_{7}\right), 4.98-5.03(2 \mathrm{H}, \mathrm{m}$, $\left.\mathrm{H}_{2}, \mathrm{H}_{4}\right), 5.89\left(1 \mathrm{H}, \mathrm{d}, J_{2,1}=4.4 \mathrm{~Hz}, \mathrm{H}_{1}\right), 5.95\left(1 \mathrm{H}\right.$, dddd, $J_{7,6}=8.8 \mathrm{~Hz}, J_{7,6}=7.6 \mathrm{~Hz}, J_{6,4}=1.8 \mathrm{~Hz}, J_{6,2}=1.8$ $\mathrm{Hz}, \mathrm{H}_{6}$ ); ${ }^{13} \mathrm{C}-\mathrm{NMR}: \delta$-5.5, -5.4, 18.2, 25.8 (3 x C), 27.7, 27.9, 32.5, 66.3, 80.3, 81.9, 105.0, 111.4, 113.2, 119.4, 147.0; Anal. Calcd for $\mathrm{C}_{17} \mathrm{H}_{29} \mathrm{NO}_{4} \mathrm{SSi}$ (371.57): C 54.95, H 7.87, N 3.77, S 8.63; found C 54.83, H 7.77, N 3.85, S 8.50.

5-O-(tert-Butyldimethylsilyl)-3-deoxy-1,2-O-isopropylidene-3-C-(Z)-(2-thiocyanatoethylidene)- $\alpha-D$ xylofuranose (6)

$\mathrm{Et}_{3} \mathrm{~N}(3.18 \mathrm{~mL}, 22.9 \mathrm{mmol})$ and $\mathrm{CH}_{3} \mathrm{SO}_{2} \mathrm{Cl}(1.42 \mathrm{~mL}, 18.3 \mathrm{mmol})$ were added at $0{ }^{\circ} \mathrm{C}$ to a solution of (Z)-4 (5.05 g, $15.3 \mathrm{mmol})$ in dry $\mathrm{CH}_{2} \mathrm{Cl}_{2}$ (36 mL). The reaction mixture was stirred for $15 \mathrm{~min}$ at $0{ }^{\circ} \mathrm{C}$ and then for $45 \mathrm{~min}$ at room temperature. The solvent was evaporated under reduced pressure. The residue was diluted with diethyl ether $(60 \mathrm{~mL})$ and the solid was removed by filtration. Evaporation of the solvent under reduced pressure afforded crude mesylate which was used directly in the next reaction without further purification. To a solution of crude mesylate (5.99 g, $14.7 \mathrm{mmol})$ in $\mathrm{CH}_{3} \mathrm{CN}$ (55 mL), KSCN (1.78 g, $18.3 \mathrm{mmol}$ ) was added. After stirring for $5 \mathrm{~h}$ at room temperature the solvent was evaporated. The residue was diluted with diethyl ether $(60 \mathrm{~mL})$ and the solid was removed by fitration. The evaporation of the solvent at reduced pressure and chromatography of the residue (hexane-ethyl acetate, 7:1) gave $4.13 \mathrm{~g}$ (73\% from 4) of pure thiocyanate 6 as a white solid; m.p. 58 $60{ }^{\circ} \mathrm{C} ;[\alpha]_{\mathrm{D}}{ }^{25}=+159.3$ (c 0.34); ${ }^{1} \mathrm{H}$ NMR: $\delta 0.06\left(3 \mathrm{H}, \mathrm{s}, \mathrm{CH}_{3}\right), 0.07\left(3 \mathrm{H}, \mathrm{s}, \mathrm{CH}_{3}\right), 0.89\left(9 \mathrm{H}, \mathrm{s}, 3 \times \mathrm{CH}_{3}\right)$, 1.41 (3H, bs, $\left.\mathrm{CH}_{3}\right), 1.46\left(3 \mathrm{H}, \mathrm{bs}, \mathrm{CH}_{3}\right), 3.66\left(1 \mathrm{H}, \mathrm{dd}, J_{5,5}=10.5 \mathrm{~Hz}, J_{5,4}=3.2 \mathrm{~Hz}, \mathrm{H}_{5}\right), 3.74(1 \mathrm{H}$, dd, $\left.J_{5,5}=10.5 \mathrm{~Hz}, J_{5,4}=4.1 \mathrm{~Hz}, \mathrm{H}_{5}\right), 3.75\left(1 \mathrm{H}\right.$, ddd, $\left.J_{7,7}=13.1 \mathrm{~Hz}, J_{7,6}=7.5 \mathrm{~Hz}, J_{7,2}=1.2 \mathrm{~Hz}, \mathrm{H}_{7}\right), 3.95(1 \mathrm{H}$, dd, $\left.J_{7,7}=13.1 \mathrm{~Hz}, J_{7,6}=8.5 \mathrm{~Hz}, \mathrm{H}_{7}\right), 4.79-4.82\left(1 \mathrm{H}, \mathrm{m}, \mathrm{H}_{4}\right), 5.13-5.15\left(1 \mathrm{H}, \mathrm{m}, \mathrm{H}_{2}\right), 5.79-5.85\left(1 \mathrm{H}, \mathrm{m}, \mathrm{H}_{6}\right)$, $5.92\left(1 \mathrm{H}, \mathrm{d}, J_{2,1}=4.5 \mathrm{~Hz}, \mathrm{H}_{1}\right)$; ${ }^{13} \mathrm{C}-\mathrm{NMR}: \delta$-5.5, -5.4, 18.2, 25.9 (3 x C), 27.6, 27.9, 32.3, 66.3, 78.9, 
81.7, 105.6, 111.7, 113.0, 119.0, 146.8; Anal. Calcd for $\mathrm{C}_{17} \mathrm{H}_{29} \mathrm{NO}_{4} \mathrm{SSi}$ (371.57): C 54.95, H 7.87, $\mathrm{N}$ 3.77, S 8.63; found C 54.86, H 7.95, N 3.85, S 8.55.

5-O-(tert-Butyldimethylsilyl)-3-deoxy-1,2-O-isopropylidene-3-isothiocyanato-3-C-vinyl- $\alpha$-D-xylofuranose (8)

A solution of $(E)-7(0.10 \mathrm{~g}, 0.27 \mathrm{mmol})$ in dry heptane $(1 \mathrm{~mL})$ was heated at $90{ }^{\circ} \mathrm{C}$ for $16 \mathrm{~h}$. The solvent was evaporated under reduced pressure and chromatography of the residue on silica gel (hexane-ethyl acetate, 13:1) afforded $0.07 \mathrm{~g}(70 \%)$ of isothiocyanate 8. Alternatively, a solution of (Z)6 (1.0 g, $2.69 \mathrm{mmol})$ in dry heptane $(5.8 \mathrm{~mL})$ was heated at $90{ }^{\circ} \mathrm{C}$ for $16 \mathrm{~h}$ under a nitrogen atmosphere. The solvent was evaporated under reduced pressure and the chromatography of the residue (hexaneethyl acetate, $13: 1)$ gave $0.82 \mathrm{~g}(82 \%)$ of isothiocyanate 8 as a colorless oil; $[\alpha]_{\mathrm{D}}{ }^{25}=+49.8(c 0.22)$; ${ }^{1} \mathrm{H}-\mathrm{NMR}: \delta 0.06\left(3 \mathrm{H}, \mathrm{s}, \mathrm{CH}_{3}\right), 0.07\left(3 \mathrm{H}, \mathrm{s}, \mathrm{CH}_{3}\right), 0.88\left(9 \mathrm{H}, \mathrm{s}, 3 \times \mathrm{CH}_{3}\right), 1.33\left(3 \mathrm{H}, \mathrm{bs}, \mathrm{CH}_{3}\right), 1.56(3 \mathrm{H}$, bs, $\left.\mathrm{CH}_{3}\right), 3.78\left(1 \mathrm{H}, \mathrm{dd}, J_{5,5}=11.1 \mathrm{~Hz}, J_{5,4}=5.5 \mathrm{~Hz}, \mathrm{H}_{5}\right), 3.86\left(1 \mathrm{H}, \mathrm{dd}, J_{5,5}=11.1 \mathrm{~Hz}, J_{5,4}=5.8 \mathrm{~Hz}, \mathrm{H}_{5}\right)$, $4.19\left(1 \mathrm{H}, \mathrm{dd}, J_{5,4}=5.8 \mathrm{~Hz}, J_{5,4}=5.5 \mathrm{~Hz}, \mathrm{H}_{4}\right), 4.50\left(1 \mathrm{H}, \mathrm{d}, J_{2,1}=3.5 \mathrm{~Hz}, \mathrm{H}_{2}\right), 5.39\left(1 \mathrm{H}, \mathrm{d}, J_{7 \mathrm{cis}, 6}=10.6 \mathrm{~Hz}\right.$, $\left.\mathrm{H}_{7 \mathrm{cis}}\right), 5.56\left(1 \mathrm{H}, \mathrm{d}, J_{7 \text { trans }, 6}=17.0 \mathrm{~Hz}, \mathrm{H}_{7 \text { trans }}\right), 5.93\left(1 \mathrm{H}, \mathrm{dd}, J_{7 \text { trans }, 6}=17.0 \mathrm{~Hz}, J_{7 \text { cis }, 6}=10.6 \mathrm{~Hz}, \mathrm{H}_{6}\right), 5.96$ $\left(1 \mathrm{H}, \mathrm{d}, J_{2,1}=3.5 \mathrm{~Hz}, \mathrm{H}_{1}\right)$; ${ }^{13} \mathrm{C}-\mathrm{NMR}: \delta$-5.5, -5.4, 18.3, 25.8 (3 x C), 26.5, 26.7, 61.1, 74.9, 82.7, 87.9, 104.3, 113.1, 118.0, 130.7, 138.2; Anal. Calcd for $\mathrm{C}_{17} \mathrm{H}_{29} \mathrm{NO}_{4} \mathrm{SSi}$ (371.57): C 54.95, H 7.87, N 3.77, S 8.63; found C 54.82, H 7.98, N 3.68, S 8.71.

5-O-(tert-Butyldimethylsilyl)-3-deoxy-1,2-O-isopropylidene-3-methoxythiocarbonylamino-3-C-vinyl$\alpha$-D-xylofuranose (9)

To a solution of isothiocyanate $8(0.58 \mathrm{~g}, 1.56 \mathrm{mmol})$ in dry methanol $(15.5 \mathrm{~mL})$ was added sodium methoxide $(0.093 \mathrm{~g}, 1.72 \mathrm{mmol})$. The reaction mixture was stirred at room temperature for $4 \mathrm{~h}$. The solvent was evaporated under reduced pressure and the residue was partitioned between $\mathrm{CH}_{2} \mathrm{Cl}_{2}$ (20 $\mathrm{mL})$ and water $(5 \mathrm{~mL})$. The organic layer was dried $\left(\mathrm{Na}_{2} \mathrm{SO}_{4}\right)$ and the solvent evaporated under reduced pressure. Chromatography of the residue (hexane-ethyl acetate, 11:1) afforded $0.60 \mathrm{~g}(95 \%)$ of compound 9 as a colorless oil; $[\alpha]_{\mathrm{D}}{ }^{25}=+62.1$ (c 0.49); ${ }^{1} \mathrm{H}-\mathrm{NMR}: \delta 0.17\left(3 \mathrm{H}, \mathrm{s}, \mathrm{CH}_{3}\right), 0.18(3 \mathrm{H}, \mathrm{s}$, $\left.\mathrm{CH}_{3}\right), 0.97$ (9H, s, $\left.3 \times \mathrm{CH}_{3}\right), 1.35$ (3H, bs, $\left.\mathrm{CH}_{3}\right), 1.52\left(3 \mathrm{H}, \mathrm{bs}, \mathrm{CH}_{3}\right), 3.71\left(1 \mathrm{H}, \mathrm{dd}, J_{5,4}=3.0 \mathrm{~Hz}, J_{5,4}=0.8\right.$ $\left.\mathrm{Hz}, \mathrm{H}_{4}\right), 3.97-4.04\left(2 \mathrm{H}, \mathrm{m}, \mathrm{H}_{5}\right), 4.01\left(3 \mathrm{H}, \mathrm{s}, \mathrm{CH}_{3} \mathrm{O}\right), 4.81\left(1 \mathrm{H}, \mathrm{d}, J_{2,1}=3.7 \mathrm{~Hz}, \mathrm{H}_{2}\right), 5.29(1 \mathrm{H}$, dd, $\left.J_{7 \text { trans }, 6}=17.5 \mathrm{~Hz}, J_{7 \text { trans,7cis }}=0.8 \mathrm{~Hz}, \mathrm{H}_{7 \text { trans }}\right), 5.33\left(1 \mathrm{H}, \mathrm{dd}, J_{7 \text { cis }, 6}=10.9 \mathrm{~Hz}, J_{7 \text { trans,7cis }}=0.8 \mathrm{~Hz}, \mathrm{H}_{7 \text { cis }}\right), 5.92$ $\left(1 \mathrm{H}, \mathrm{d}, J_{2,1}=3.7 \mathrm{~Hz}, \mathrm{H}_{1}\right), 6.01\left(1 \mathrm{H}, \mathrm{dd}, J_{7 \text { trans }, 6}=17.5 \mathrm{~Hz}, J_{7 \mathrm{cis}, 6}=10.9 \mathrm{~Hz}, \mathrm{H}_{6}\right), 9.37(1 \mathrm{H}, \mathrm{bs}, \mathrm{NH}) ;{ }^{13} \mathrm{C}-$ NMR: $\delta$-5.6, -5.3, 18.5, 26.0 (3 x C), 26.4, 26.7, 57.9, 59.1, 71.6, 78.6., 83.7, 104.5, 112.2, 117.0, 131.9, 191.3; Anal. Calcd for $\mathrm{C}_{18} \mathrm{H}_{33} \mathrm{NO}_{5} \mathrm{SSi}$ (403.62): C 53.57, H 8.24, N 3.47, S 7.94; found C 53.80, H 8.38, N 3.66, S 7.70.

5-O-(tert-Butyldimethylsilyl)-3-deoxy-1,2-O-isopropylidene-3-methoxycarbonylamino-3-C-vinyl- $\alpha$ - $D$ xylofuranose (10)

To a solution of 9 (0.56 g, $1.39 \mathrm{mmol})$ in $\mathrm{CH}_{3} \mathrm{CN}(13.5 \mathrm{~mL})$ was added mesitylnitrile oxide (0.25 g, $1.53 \mathrm{mmol})$. The reaction mixture was stirred at room temperature for $23 \mathrm{~h}$, acetonitrile was 
evaporated under reduce pressure. Chromatography of residue (hexane-ethyl acetate, 9:1) gave $0.50 \mathrm{~g}$ (93\%) of 10 as a white crystals; m.p. $103-106{ }^{\circ} \mathrm{C}$; $[\alpha]_{\mathrm{D}}{ }^{25}=+69.5$ (c 0.28); ${ }^{1} \mathrm{H}-\mathrm{NMR}$ : $\delta 0.11$ (3H, s, $\mathrm{CH}_{3}$ ), 0.12 (3H, s, $\mathrm{CH}_{3}$ ), 0.92 (9H, s, 3 x CH $\left.\mathrm{CH}_{3}\right), 1.33$ (3H, bs, $\left.\mathrm{CH}_{3}\right), 1.52$ (3H, bs, $\left.\mathrm{CH}_{3}\right), 3.62(3 \mathrm{H}, \mathrm{s}$, $\left.\mathrm{CH}_{3} \mathrm{O}\right), 3.79\left(1 \mathrm{H}, \mathrm{m}, \mathrm{H}_{4}\right), 3.94\left(1 \mathrm{H}, \mathrm{d}, J_{5,5}=12.4 \mathrm{~Hz}, \mathrm{H}_{5}\right), 4.06\left(1 \mathrm{H}, \mathrm{dd}, J_{5,5}=12.4 \mathrm{~Hz}, J_{5,4}=3.0 \mathrm{~Hz}, \mathrm{H}_{5}\right)$, $5.07\left(1 \mathrm{H}, \mathrm{d}, J_{2,1}=3.5 \mathrm{~Hz}, \mathrm{H}_{2}\right), 5.32\left(1 \mathrm{H}, \mathrm{d}, J_{7 \text { trans }, 6}=17.5 \mathrm{~Hz}, \mathrm{H}_{7 \text { trans }}\right), 5.37\left(1 \mathrm{H}, \mathrm{d}, J_{7 \mathrm{cis}, 6}=10.8 \mathrm{~Hz}, \mathrm{H}_{7 \mathrm{cis}}\right)$, $5.88\left(1 \mathrm{H}, \mathrm{d}, J_{2,1}=3.5 \mathrm{~Hz}, \mathrm{H}_{1}\right), 6.08\left(1 \mathrm{H}\right.$, dd, $\left.J_{7 \text { trans }, 6}=17.5 \mathrm{~Hz}, J_{7 \mathrm{cis}, 6}=10.8 \mathrm{~Hz}, \mathrm{H}_{6}\right), 7.57(1 \mathrm{H}, \mathrm{bs}, \mathrm{NH})$; ${ }^{13}$ C-NMR: $\delta$-5.7, -5.5, 18.2, 25.7 (3 x C), 26.3, 26.8, 51.8, 59.4, 68.6, 78.7., 83.4, 104.5, 112.1, 116.6, 132.6, 155.8; Anal. Calcd for $\mathrm{C}_{18} \mathrm{H}_{33} \mathrm{NO}_{6} \mathrm{Si}$ (387.55): C 55.79, $\mathrm{H}$ 8.58, N 3.61; found C 55.58, H 8.27, N 3.42 .

5-O-(tert-Butyldimethylsilyl)-3-deoxy-1,2-O-isopropylidene-3-methoxycarbonylamino- $\alpha$-D-xylofuranose 3-C-carbaldehyde (11)

To a solution of $\mathbf{1 0}(0.30 \mathrm{~g}, 0.77 \mathrm{mmol})$ in 2:2:3 $\mathrm{CCl}_{4} / \mathrm{CH}_{3} \mathrm{CN} / \mathrm{H}_{2} \mathrm{O}(8.5 \mathrm{~mL})$ were added sodium periodate $(0.68 \mathrm{~g}, 3.16 \mathrm{mmol})$ and ruthenium trichloride hydrate $(4.2 \mathrm{mg}, 2.5 \mathrm{~mol} \%)$. The reaction mixture was stirred at room temperature for $20 \mathrm{~h}$, then extracted with $\mathrm{CH}_{2} \mathrm{Cl}_{2}(3 \mathrm{x} 20 \mathrm{~mL}$ ). The combined organic layers were dried $\left(\mathrm{Na}_{2} \mathrm{SO}_{4}\right)$ and concentrated under reduced pressure. The residue was purified by chromatography (hexane-ethyl acetate, 7:1) to afford $0.21 \mathrm{~g}$ (70\%) of compound 11 as a colorless oil; $[\alpha]_{\mathrm{D}}{ }^{25}=+69.3\left(c\right.$ 0.29); ${ }^{1} \mathrm{H}-\mathrm{NMR}: \delta 0.09\left(3 \mathrm{H}, \mathrm{s}, \mathrm{CH}_{3}\right), 0.10\left(3 \mathrm{H}, \mathrm{s}, \mathrm{CH}_{3}\right), 0.90(9 \mathrm{H}, \mathrm{s}, 3$ x $\left.\mathrm{CH}_{3}\right), 1.35$ (3H, bs, $\left.\mathrm{CH}_{3}\right), 1.56\left(3 \mathrm{H}, \mathrm{bs}, \mathrm{CH}_{3}\right), 3.67\left(3 \mathrm{H}, \mathrm{s}, \mathrm{CH}_{3} \mathrm{O}\right), 3.92\left(1 \mathrm{H}, \mathrm{dd}, J_{5,5}=12.1 \mathrm{~Hz}\right.$, $\left.J_{5,4}=1.8 \mathrm{~Hz}, \mathrm{H}_{5}\right), 4.01\left(1 \mathrm{H}, \mathrm{dd}, J_{5,5}=12.1 \mathrm{~Hz}, J_{5,4}=4.2 \mathrm{~Hz}, \mathrm{H}_{5}\right), 4.27\left(1 \mathrm{H}, \mathrm{dd}, J_{5,4}=4.2 \mathrm{~Hz}, J_{5,4}=1.8 \mathrm{~Hz}\right.$, $\left.\mathrm{H}_{4}\right) 5.12\left(1 \mathrm{H}, \mathrm{d}, J_{2,1}=3.6 \mathrm{~Hz}, \mathrm{H}_{2}\right), 5.99\left(1 \mathrm{H}, \mathrm{d}, J_{2,1}=3.6 \mathrm{~Hz}, \mathrm{H}_{1}\right), 7.31(1 \mathrm{H}, \mathrm{bs}, \mathrm{NH}), 9.91(1 \mathrm{H}, \mathrm{s}, \mathrm{CHO})$; ${ }^{13}$ C-NMR: $\delta$-5.7, -5.6, 18.1, 25.7 (3 x C), 26.3, 26.7, 52.4, 60.3, 71.8, 76.2, 84.4, 105.3, 113.3, 156.5, 197.4; Anal. Calcd for $\mathrm{C}_{17} \mathrm{H}_{31} \mathrm{NO}_{7} \mathrm{Si}$ (389.53): C 52.42, H 8.02, N 3.60; found C 52.27, H 8.27, N 3.42.

5-O-(tert-Butyldimethylsilyl)-3-deoxy-1,2-O-isopropylidene-3-methoxycarbonylamino- $\alpha$-D-xylofuranose 3-C-carboxylic acid (12)

A solution of $\mathrm{NaClO}_{2}(0.23 \mathrm{~g}, 2.54 \mathrm{mmol})$ and $\mathrm{NaH}_{2} \mathrm{PO}_{4}(0.285 \mathrm{~g}, 1.83 \mathrm{mmol})$ in water $(1.55 \mathrm{~mL})$ was added dropwise to the solution of aldehyde 11 (0.107 g, $0.275 \mathrm{mmol})$ in 4:4:1 acetonitrile/tertbutyl alcohol/2-methyl-2-butene $(6.2 \mathrm{~mL})$ at $0{ }^{\circ} \mathrm{C}$ over $5 \mathrm{~min}$ and then stirred at the same temperature for $25 \mathrm{~min}$. The reaction mixture was poured into brine $(8 \mathrm{~mL})$ and extracted with ethyl acetate $(3 \times 10$ $\mathrm{mL})$. The combined organic layers were dried $\left(\mathrm{Na}_{2} \mathrm{SO}_{4}\right)$ and concentrated under reduced pressure. The residue was purified by chromatography (1:2 hexane-ethyl acetate) to give $0.082 \mathrm{~g}$ (74\%) of carboxylic acid 12 as a colorless oil; $[\alpha]_{\mathrm{D}}{ }^{25}=+45.4$ (c 0.57); ${ }^{1} \mathrm{H}-\mathrm{NMR}: \delta 0.11\left(3 \mathrm{H}, \mathrm{s}, \mathrm{CH}_{3}\right), 0.12(3 \mathrm{H}, \mathrm{s}$, $\left.\mathrm{CH}_{3}\right), 0.91$ (9H, s, $\left.3 \times \mathrm{CH}_{3}\right), 1.35$ (3H, bs, $\left.\mathrm{CH}_{3}\right), 1.53$ (3H, bs, $\left.\mathrm{CH}_{3}\right), 3.76$ (3H, s, $\left.\mathrm{CH}_{3} \mathrm{O}\right), 4.22(2 \mathrm{H}, \mathrm{m}$, $\left.\mathrm{H}_{5}\right), 4.42\left(1 \mathrm{H}, \mathrm{m}, \mathrm{H}_{4}\right), 5.04\left(1 \mathrm{H}, \mathrm{d}, J_{2,1}=3.9 \mathrm{~Hz}, \mathrm{H}_{2}\right), 5.95\left(1 \mathrm{H}, \mathrm{d}, J_{2,1}=3.9 \mathrm{~Hz}, \mathrm{H}_{1}\right), 8.30(1 \mathrm{H}, \mathrm{bs}, \mathrm{NH})$; ${ }^{13}$ C-NMR: $\delta$-5.7, -5.6, 18.2, 25.6 (3 x C), 26.3, 26.5, 53.4, 61.0, 70.6, 76.8, 82.1, 104.3, 113.2, 159.5, 167.6; Anal. Calcd for $\mathrm{C}_{17} \mathrm{H}_{31} \mathrm{NO}_{8} \mathrm{Si}$ (405.52): C 50.35, H 7.71, N 3.45; found C 50.17, H 7.53, N 3.20. 
5-O-[(tert-Butyldimethylsilyl)-3-deoxy-1,2-O-isopropylidene-3-methoxycarbonylamino- $\alpha$ - $D$-xylofuranosyl-3-C-carbonyl]glycine methyl ester (13)

To a solution of glycine methyl ester hydrochloride $(11 \mathrm{mg}, 0.0886 \mathrm{mmol})$ in dry dichloromethane $(0.5 \mathrm{~mL})$ was added $\mathrm{Et}_{3} \mathrm{~N}(0.041 \mathrm{~mL}, 0.30 \mathrm{mmol})$. The suspension was cooled to $0{ }^{\circ} \mathrm{C}$. Then, a solution of aminoacid 12 (24 mg, $0.059 \mathrm{mmol})$ in dry $\mathrm{CH}_{2} \mathrm{Cl}_{2}(0.3 \mathrm{~mL})$ and DCC (24.4 mg, $\left.0.12 \mathrm{mmol}\right)$ were added. The reaction mixture was stirred at $0{ }^{\circ} \mathrm{C}$ for $1 \mathrm{~h}$ and then at room temperature for $18 \mathrm{~h}$. Dichloromethane $(3 \mathrm{~mL})$ was added and solution was washed with ice water $(0.5 \mathrm{~mL})$. The organic layer was dried $\left(\mathrm{Na}_{2} \mathrm{SO}_{4}\right)$ and concentrated under reduced pressure. The residue was purified by chromatography (2:1 hexane-ethyl acetate) to afford $22 \mathrm{mg}$ (78\%) of dipeptide $\mathbf{1 3}$ as a colorless oil; $[\alpha]_{\mathrm{D}}{ }^{25}=+35.1$ (c 0.14); ${ }^{1} \mathrm{H}-\mathrm{NMR}: \delta 0.11\left(3 \mathrm{H}, \mathrm{s}, \mathrm{CH}_{3}\right), 0.12\left(3 \mathrm{H}, \mathrm{s}, \mathrm{CH}_{3}\right), 0.91\left(9 \mathrm{H}, \mathrm{s}, 3 \times \mathrm{CH}_{3}\right), 1.37$ (3H, bs, $\left.\mathrm{CH}_{3}\right), 1.58\left(3 \mathrm{H}, \mathrm{bs}, \mathrm{CH}_{3}\right), 3.67\left(3 \mathrm{H}, \mathrm{s}, \mathrm{CH}_{3} \mathrm{O}\right), 3.75\left(3 \mathrm{H}, \mathrm{s}, \mathrm{CH}_{3} \mathrm{O}\right), 4.01(1 \mathrm{H}, \mathrm{dd}, \mathrm{J}=18.4 \mathrm{~Hz}$, $\left.J_{\mathrm{CH} 2, \mathrm{NH}}=4.7 \mathrm{~Hz}, \mathrm{CH}_{2} \mathrm{NH}\right), 4.13\left(1 \mathrm{H}, \mathrm{dd}, J_{5,5}=12.4 \mathrm{~Hz}, J_{5,4}=2.8 \mathrm{~Hz}, \mathrm{H}_{5}\right), 4.18\left(1 \mathrm{H}, \mathrm{m}, \mathrm{H}_{5}\right), 4.19(1 \mathrm{H}$, dd, $\left.J=18.4 \mathrm{~Hz}, J_{\mathrm{CH} 2, \mathrm{NH}}=5.7 \mathrm{~Hz}, \mathrm{CH}_{2} \mathrm{NH}\right), 4.25\left(1 \mathrm{H}, \mathrm{m}, \mathrm{H}_{4}\right), 5.15\left(1 \mathrm{H}, \mathrm{d}, J_{2,1}=3.6 \mathrm{~Hz}, \mathrm{H}_{2}\right), 5.92(1 \mathrm{H}, \mathrm{d}$, $\left.J_{2,1}=3.6 \mathrm{~Hz}, \mathrm{H}_{1}\right), 7.93\left(1 \mathrm{H}\right.$, bs, NH), $8.28\left(1 \mathrm{H}, \mathrm{dd}, J_{\mathrm{CH} 2, \mathrm{NH}}=5.7 \mathrm{~Hz}, J_{\mathrm{CH} 2, \mathrm{NH}}=4.7 \mathrm{~Hz}, \mathrm{NH}\right) ;{ }^{13} \mathrm{C}-\mathrm{NMR}: \delta$ -5.7, -5.6, 18.2, 25.7 (3 x C), 26.5, 26.8, 41.5, 52.2, 52.3, 61.0, 69.7, 78.2, 82.6, 104.9, 113.1, 157.2, 168.3, 170.1; Anal. Calcd for $\mathrm{C}_{20} \mathrm{H}_{36} \mathrm{~N}_{2} \mathrm{O}_{9} \mathrm{Si}$ (476.60): C 50.40, H 7.61, N 5.88; found C 50.22, H 7.49, N 5.61 .

\section{Acknowledgements}

This work was supported by the Grant Agency (No.1/2472/05) of the Ministry of Education, Slovak Republic. NMR experiments were supported by Establishment of the "top-class” laboratory for Nuclear Magnetic Resonance (No. 200280203/2003) of the Ministry of Education, Slovak Rebublic.

\section{References and Notes}

1. (a) Lohof, E.; Burkhart, F.; Born, M. A.; Planker, E.; Kesler, H. In Advances in Amino Acid Mimetics and Peptidomimetics; Abell, A., Ed.; JAI Press Inc: Stanford, Connecticut, 1999; Vol. 2, p. 263; (b) Schweizer, F. Glycosamino Acids: Building Blocks for Combinatorial SynthesisImplications for Drug Discovery. Angew. Chem. Int. Ed. 2002, 41, 230-253; (c) Gruner, S. A. V.; Locardi, E.; Lohof, E.; Kessler, H. Carbohydrate-Based Mimetics in Drug Design: Sugar Amino Acids and Carbohydrate Scaffolds. Chem. Rev. 2002, 102, 491-514.

2. (a) Graf von Roedern, E.; Lohof, E.; Hessler, G.; Hoffmann, M.; Kessler, H. Synthesis and Conformational Analysis of Linear and Cyclic Peptides Containg Amino Acids. J. Am. Chem. Soc. 1996, 118, 10156-10167; (b) Le Tiran, A.; Stables, J. P.; Kohn, H. Functionalized Amino Acid Anticonvulsants: Synthesis and Pharmacological Evaluation of Conformationally Restricted Analogues. Bioorg. Med. Chem. 2001, 9, 2693-2708; (c) Chakraborty, T. K.; Jayaprakash, S.; Srinivasu, P.; Madhavendra, S. S.; Ravi Sankar, A.; Kunwar, A. C. Furanoid sugar amino acid based peptidomimetics: well-defined solution conformations to gel-like structures. Tetrahedron 2002, 58, 2853-2859. 
3. (a) Chakraborty, T. K; Jayaprakash, S.; Diwan, P. V.; Nagaraj, R.; Jampani, S. R. B.; Kunwar, A. C. Folded Conformation in Peptide Containing Furanoid Sugar Amino Acids. J. Am. Chem. Soc. 1998, 120, 12962-12963; (b) Dondoni, A.; Marra, A. Methods for Anomeric Carbon-linked and Fused Sugar Amino Acid Synthesis: The Gateway to Artificial Glycopeptides. Chem. Rev. 2000, 100, 4395-4421; (c) Locardi, E.; Stöckle, M.; Gruner, S.; Kessler, H. Cyclic Homooligomers from Sugar Amino Acids: Synthesis, Conformational Analysis, and Significance. J. Am. Chem. Soc. 2001, 123, 8189-8196; (d) De Borggraeve, W. M.; Rombouts, F. J. R.; Van der Eycken, E. V.; Toppet, S. M.; Hoornaert, G. J. Synthesis of a conformationally restricted dipeptide analogue and its evaluation as a $\beta$-turn mimic. Tetrahedron Lett. 2001, 42, 5693-5695; (e) Nguyen Van Nhien, A.; Ducatel , H.; Len, Ch.; Postel, D. Novel conformationally restricted glycoamino acids from glyco- $\alpha$-aminonitriles as potent turn mimics in peptide synthesis. Tetrahedron Lett. 2002, 43, 3805-3808; (f) McGarvey, G. J.; Benedum, T. E.; Schmidtmann, F. W. Development of Co- and Post-Translational Synthetic Strategies to C-Neoglycopeptides. Org. Lett. 2002, 4, 3591-3594; (g) Xie, J. Synthesis of new sugar amino acid derivatives of D-glucosamine. Carbohydr. Res. 2003, 338, 399-406; (h) Billing, J. F.; Nilsson, U. J. Cyclic peptides containig a $\delta$-sugar amino acidsynthesis and evaluation as artificial receptors. Tetrahedron 2005, 61, 863-874; (i) Simone, M. I.; Soengas, R.; Newton, Ch. R.; Watkin, D. J.; Fleet, G. W. J. Branched tetrahydrofuran $\alpha, \alpha-$ disubstitued- $\delta$-sugar amino acid scaffolds from branched sugar lactones: a new family of foldamers? Tetrahedron Lett. 2005, 46, 5761-5765; (j) Schmidtmann, F. W.; Benedum, T. E.; McGarvey, G. $J$. The preparation of $C$-glycosyl amino acids - an examination of olefin crossmetathesis. Tetrahedron Lett. 2005, 46, 4677-4681; (k) Schweizer, F; Hindsgaul, O. Synthesis of a galacto-configured C-ketoside-based $\gamma$-sugar-amino acid and its use in peptide coupling reactions. Carbohydr. Res. 2006, 341, 1730-1736.

4. Lu, Y.; Just, G. Stereoselective synthesis of dithymidine phosphorothioates using D-xylose derived chiral auxiliaries. Tetrahedron 2001, 57, 1677-1687.

5. Podlech, J. In Houben-Weyl, Bd.; Thieme: New York, 2001; Vol. E 22a, pp. 517-533.

Sample Availability: Samples of the compounds (Z)-6, 8, 10 are available from the authors.

(c) 2006 by MDPI (http://www.mdpi.org). Reproduction is permitted for noncommercial purposes. 